\title{
Raman scattering and photoluminescence analysis of silicon on insulator structures obtained by single and multiple oxygen implants
}

\author{
A. Pérez-Rodríguez, A. Cornet, and J. R. Morante \\ LCMM, Departament de Fisica Aplicada i Electrònica, Universitat de Barcelona, Avda. Diagonal 645-647, \\ 08028-Barcelona, Spain \\ J. Jimenez \\ Departamento de Fisica de la Materia Condensada, Universidad de Valladolid, 47011-Valladolid, Spain \\ P. L. F. Hemment and K. P. Homewood \\ Department of Electronic and Electrical Engineering, University of Surrey, Guildford, Surrey GU2 $5 X H$, \\ United Kingdom
}

(Received 27 December 1990; accepted for publication 25 April 1991)

\begin{abstract}
An analysis of silicon on insulator structures obtained by single and multiple implants by means of Raman scattering and photoluminescence spectroscopy is reported. The Raman spectra obtained with different excitation powers and wavelengths indicate the presence of a tensile strain in the top silicon layer of the structures. The comparison between the spectra measured in both kinds of samples points out the existence in the multiple implant material of a lower strain for a penetration depth about $300 \mathrm{~nm}$ and a higher strain for higher penetration depths. These results have been correlated with transmission electron microscopy observations, which have allowed to associate the higher strain to the presence of $\mathrm{SiO}_{2}$ precipitates in the top silicon layer, close to the buried oxide. The found lower strain is in agreement with the better quality expected for this material, which is corroborated by the photoluminescence data.
\end{abstract}

\section{INTRODUCTION}

Silicon on insulator (SOI) technology represents one of the more interesting alternatives to bulk silicon for the production of the substrate material for very large scale integration (VLSI) applications. ${ }^{1}$ Between the different methods of making SOI material, Separation by Implanted Oxygen (SIMOX) appears to be the leading one. SIMOX material is prepared by a high dose $\left(>10^{18} \mathrm{~cm}^{-2}\right)$ oxygen implantation followed by a high temperature anneal (HTA) stage ( $T \geqslant 1300^{\circ} \mathrm{C}$ for several hours). The quality of the structures produced by this method has allowed the achievement of high performance small geometry CMOS devices showing significant improvements over similar circuits fabricated on bulk silicon. ${ }^{1,2}$

Problems related to this technology are basically due to the presence of a high concentration of defects remaining in both the top silicon and the buried oxide layers after the anneal. ${ }^{1}$ Defects in the top silicon layer are mainly dislocations. Although they seem to cause not considerable degradation of MOS devices and circuit performance, their presence is highly undesirable as they are a potential cause of circuit failure and represent a grave limitation for the application of the material, especially for bipolar devices. Defects in the buried oxide layer are basically silicon islands located in bands near the upper and the lower $\mathrm{Si}-\mathrm{SiO}_{2}$ interfaces of the $\mathrm{SiO}_{2}$ layer. The appearance of one band (close to the lower interface) or two bands of $\mathrm{Si}$ islands depends on the implanted dose. ${ }^{3}$ Once formed, these islands are stable and difficult to disperse. They do not seem to affect the device characteristics greatly, although they cause a reduction of the breakdown strength of the buried oxide and may modify its radiation hardness properties. Moreover, previous analysis by Raman spectroscopy of these structures has also revealed the existence of tensile strains in the top silicon layer, which affect device performance. ${ }^{4}$

In order to resolve these problems, different alternative methods of obtaining SIMOX material have been proposed. Between them, a very promising one is sequential implantation and anneal (SIA). ${ }^{4}$ In this method, the structures are obtained by multiple low dose implantations followed by HTA stages. Many authors have already reported a significant reduction of the defect density in SIMOX structures obtained by SIA when compared with those obtained by a standard, single implant and anneal (which will be referred as the SS process).$^{5-7}$ However, the mechanisms related to the defects present in the structures and their dependence on the technological parameters are not well known. This has lead to strong interest in the characterization of this kind of structures for the optimization of the processing parameters needed to achieve reproducible low defect density material with strain free silicon layers.

In this work we have analyzed the strain and defects present in SIMOX structures obtained by sequential implantation and annealing by means of Raman scattering and photoluminescence (PL) spectroscopy. This has been done in a comparative way, in relation to equivalent structures obtained by standard SS processing. Raman scattering and PL techniques have a special interest due to their nondestructive character as well as their suitability for the study of thin layered structures typical of SOI materials. ${ }^{8,9}$

Raman spectra have been obtained using different excitation powers and wavelengths, in order to investigate the dependence of the spectra on the temperature of the scat- 
TABLE I. Process conditions of the SIMOX samples.

\begin{tabular}{lccccc}
\hline \hline $\begin{array}{l}\text { Spec. } \\
\text { No. }\end{array}$ & $E(\mathrm{keV})$ & $\phi\left(\mathrm{cm}^{-2}\right)$ & $T_{\text {imp }}\left({ }^{\circ} \mathrm{C}\right)$ & $T_{a}\left({ }^{\circ} \mathrm{C}\right)$ & time (h) \\
\hline 1 SS & 200 & $1.7 \times 10^{18}$ & 640 & 1300 & 6 \\
2 SIA & 200 & $3 \times\left(5 \times 10^{17}\right)$ & 640 & 1300 & $3 \times 2$ \\
\hline
\end{tabular}

tering region, for different penetration depths. The observation of the samples by cross section transmission electron microscopy (XTEM) has allowed us to correlate this data with their microscopic structure and to determine the contribution of each region of the structures to the spectra. Their comparison with the spectra corresponding to bulk silicon indicates how much lattice strain is present in the different regions, which provides information relevant for the analysis of the transport properties of the devices built in these materials. The correlation of this data with that obtained from XTEM and PL techniques has allowed us to characterize the different thin films in the structures, observing the existence of significant differences related to the technological process (SS or SIA).

\section{EXPERIMENT}

SIMOX wafers were produced by Ibis Technology Corp. ${ }^{10}$ Two different sets of samples were measured which, according to their preparation, were labelled SS and SIA as listed in Table I. The SS samples were made by a single step oxygen implantation at an energy of $200 \mathrm{keV}$ and a dose of $1.7 \times 10^{18} \mathrm{~cm}^{-2}$. During implantation the substrate was held at a temperature of $640^{\circ} \mathrm{C}$. Subsequently, samples were annealed at a temperature of $1300^{\circ} \mathrm{C}$. The SIA samples were obtained by implanting one third of the total dose at $640^{\circ} \mathrm{C}$ after which the wafer was annealed at $1300^{\circ} \mathrm{C}$ for $2 \mathrm{~h}$. This cycle was carried out three times to give a total dose of $1.5 \times 10^{18} \mathrm{~cm}^{-2}$ and a total annealing time of 6 hours at $1300^{\circ} \mathrm{C}$.

Samples have been observed by XTEM with a $200-\mathrm{keV}$ microscope, a Hitachi NA-800, which has a resolution of $2.7 \AA$. Cross section samples were prepared by gluing, dimpling and ion milling of $3 \mathrm{~mm} \times 350 \mu \mathrm{m}$ pieces of the wafers. ${ }^{11}$ This observation shows the presence of defects in the studied structures, basically, $\mathrm{Si}$ islands in the $\mathrm{SiO}_{2}$ buried layer of SS samples and $\mathrm{SiO}_{2}$ precipitates and dislocations close to the upper $\mathrm{Si} /$ buried $\mathrm{SiO}_{2}$ interface of SIA samples. Therefore, SS and SIA samples do not correspond to optimized structures for the device's realization. However, the analysis of such samples by optical techniques such as Raman scattering and photoluminescence has a strong interest, as it allows us to determine the role played by these defects on the physical characteristics of the different layers composing the structure, mainly on the strain present in the layers.

Raman scattering measurements have been performed with a DILOR XY spectrometer. The samples were excited with the 457.9, 488, and 514-nm lines from an $\mathrm{Ar}^{+}$laser, with different powers through microscope optics in backscattering configuration. For these lines, the

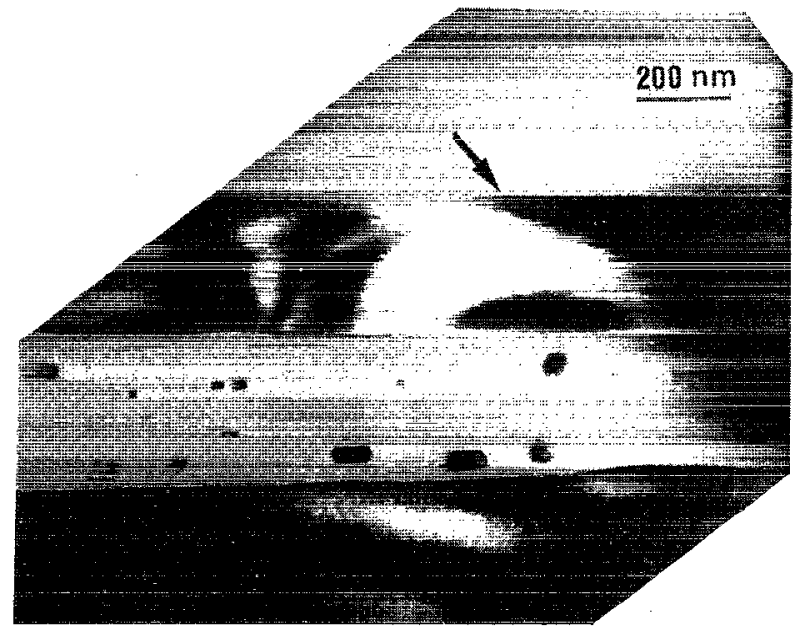

FIG. 1. Cross-section image of sample SS. The arrow indicates the upper surface.

penetration depths for the total scattered light have been estimated to be of approximately 300,600 , and $800 \mathrm{~nm}$, respectively. ${ }^{12}$ Detection has been made using multichannel mode with photon counting electronics. The position of the Raman peaks has been measured with an accuracy of $0.3 \mathrm{~cm}^{-1}$ and the linewidths with a spectral resolution better than $1.5 \mathrm{~cm}^{-1}$. The system was calibrated before and after each measurement, in order to correct frequency shifts. The calibration was made with different lines from a Ne lamp.

Finally, photoluminescence measurements have been made over the energy range 0.750 to $1.160 \mathrm{eV}$. Samples were also excited with the $457.9,488$, and $514 \mathrm{~nm} \cdot$ lines from the $\mathrm{Ar}^{+}$laser, the power used being $0.5 \mathrm{~W} / \mathrm{cm}^{2}$. For this value of nominal power, the temperature of the samples has been estimated to be $25 \mathrm{~K}$. Their spectral response was determined with a grating monochromator (JobinYvon, model HR2) with a resolution of $13 \AA / \mathrm{mm}$, using a cooled InAs detector and a conventional lock-in amplifier. The spectra were normalized to compensate for the detector response.

\section{RESULTS AND DISCUSSION}

The cross-section structure of the SS samples is shown in Fig. 1. It is formed by a top silicon layer free of $\mathrm{SiO}_{2}$ precipitates and a buried oxide layer with silicon islands. The interfaces between the buried layer and both the top $\mathrm{Si}$ and the substrate are sharp. These layers have thicknesses between 280 and $310 \mathrm{~nm}$ (top $\mathrm{Si}$ ) and between 290 and 330 $\mathrm{nm}$ (buried $\mathrm{SiO}_{2}$ ), observed along $2 \mu \mathrm{m}$ of the structure. These differences seem to be partly due to the existence of oscillations of both interfaces, but mainly of the upper one (up to $45 \mathrm{~nm}$ ). Moreover, the oxide layer is characterized by two bands of $\mathrm{Si}$ islands which appear at the upper and lower half of the oxide, respectively. They are about 20 to $55 \mathrm{~nm}$ thick and 25 to $115 \mathrm{~nm}$ long, with well developed facets along the $\{100\}$ and $\{111\}$ planes. They appear at an average distance of about $100 \mathrm{~nm}$ and $20 \mathrm{~nm}$ from the 


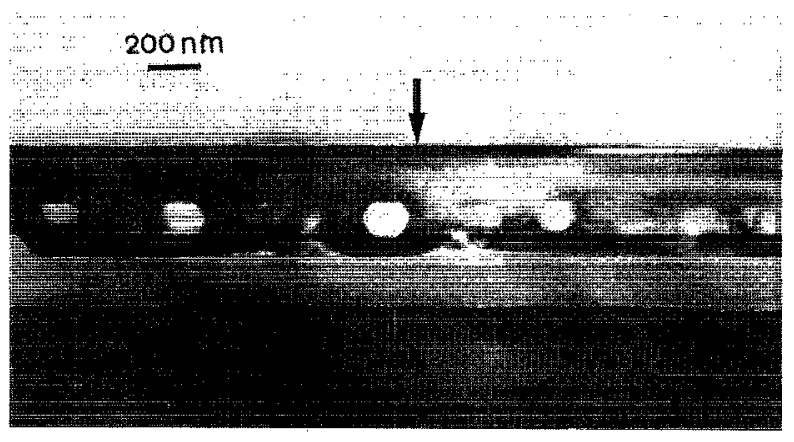

FIG. 2. Cross-section image of sample SIA. The arrow indicates the upper surface.

respective interfaces. In general, islands at the upper half of the oxide are smaller.

Figure 2 shows a cross section image of the SIA samples. In this case, the structure presents three well-defined regions. The first one is a layer of high quality $\mathrm{Si}$ with small thickness variation ( 233 to $240 \mathrm{~nm}$ ) and free of precipitates and dislocations. Afterwards there is a layer of $\mathrm{Si}$ with $\mathrm{SiO}_{2}$ precipitates. These precipitates tend to have polihedrical shapes, with well defined facets along the $\{100\}$ and $\{111\}$ planes. They are 95 to $180 \mathrm{~nm}$ thick, at a distance between 20 and $70 \mathrm{~nm}$ from the $\mathrm{SiO}_{2}$ buried layer. This layer constitutes the third region. It appears free of $\mathrm{Si}$ islands, with a very wavy upper interface. This gives a $\mathrm{SiO}_{2}$ thickness between 180 and $270 \mathrm{~nm}$. However, the thickness of the total structure $\left(\mathrm{Si}+\mathrm{SiO}_{2}\right)$ shows a very small spread ( 644 to $652 \mathrm{~nm}$ ). The waviness of the upper interface is due to the coalescence of $\mathrm{SiO}_{2}$ precipitates in the buried layer. Morcover, the precipitates region is characterized by a high density of dislocations connecting the precipitates between them and with the buried oxide layer, as is shown in Fig. 3. However, we have not observed any dislocations running towards the upper surface.

As it has been already indicated, SIA method has been shown to give low defect density, planar SIMOX struc-

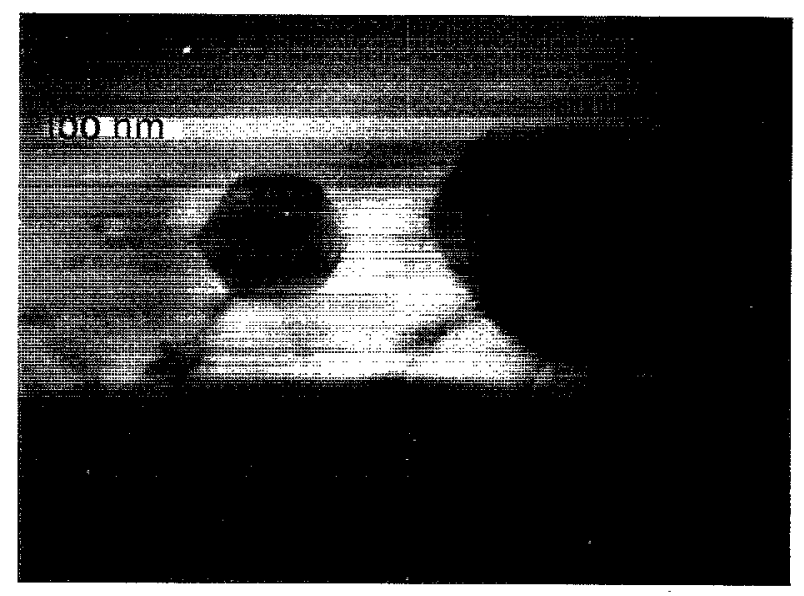

FIG. 3. Dark field image of the precipitates region in the SIA sample, showing dislocations pinned in this region.

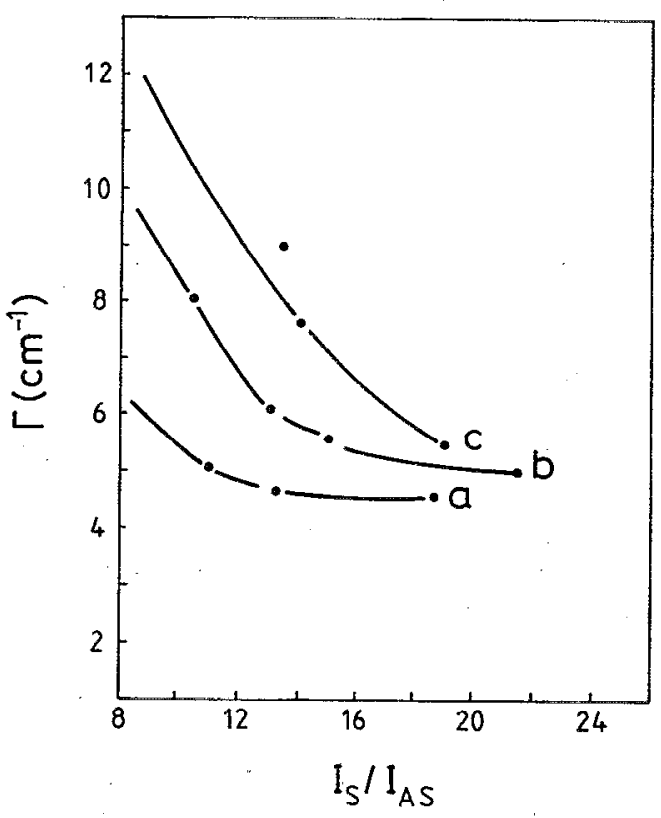

FIG. 4. Broadening of the first-order Raman lines vs Stokes to anti-Stokes intensity ratio for (a) bulk Si, (b) SS and (c) SIA samples. Excitation wavelength $514 \mathrm{~nm}$.

tures. ${ }^{4}$ However, the processing conditions for the SIA wafer used in this experiment were not optimized and the structure shown in Fig. 2 is not typical of SIA material. The wafers (SS and SIA, Table I) were chosen to show the high sensitivity and versatility of the optical analysis methods used in this work.

As the temperature gradient in the samples due to their heating by the excitation laser can determine both a shift and a broadening of the Raman lines which depend on the sample characteristics, Raman scattering measurements have been made using different excitation powers and wavelengths. In Fig. 4 we have plotted the linewidth of the Raman peaks measured in the different samples (SS, SIA and bulk silicon) versus the Stokes to antistokes intensity ratios (which depend on the temperature of the scattering volume) for different excitation powers and an excitation wavelength of $514 \mathrm{~nm}$ (penetration depth about $800 \mathrm{~nm}$ ). Always the peaks obtained in SS or SIA samples are broader than those measured in bulk silicon under the same temperature conditions. This behavior is found for all the excitation wavelengths and indicates that the broadening observed in the Raman peaks are not only due to a tempcrature effect, but they are also dependent on the sample response to such a temperature effect. So, both SIA and SS samples clearly show a very different behavior than that of bulk silicon as the temperature of the scattering volume increases. It has to be noted that at low excitation powers, similar to those used in previous works, ${ }^{12,13}$ this behavior is scarcely observed. The temperature in the laser spot has been estimated to be lower than $150^{\circ} \mathrm{C}$, for the higher excitation power, which determines a temperature gradient higher than $100^{\circ} \mathrm{C} / \mu \mathrm{m}$.

Figure 5 shows the spectra obtained for the different 


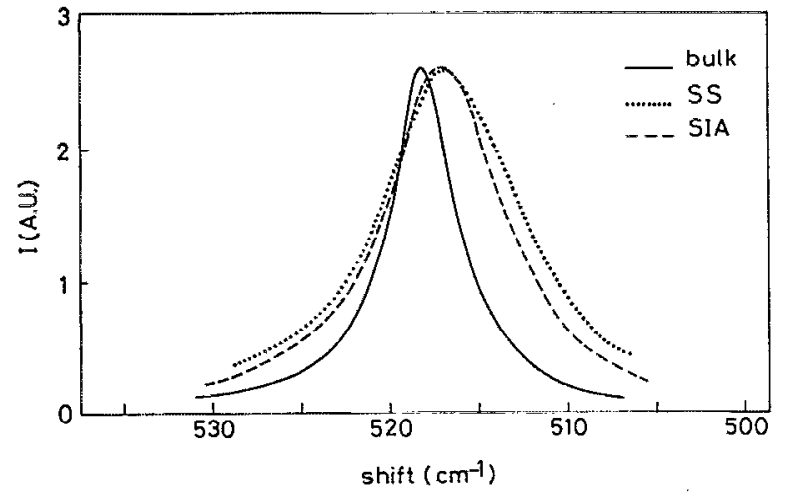

FIG. 5. First-order Raman spectra obtained from the different samples with an excitation power of $0.5 \mathrm{MW} / \mathrm{cm}^{2}$ and wavelength $457.9 \mathrm{~nm}$.

samples with an excitation wavelength of $457.9 \mathrm{~nm}$ and a power of $0.5 \mathrm{MW} / \mathrm{cm}^{2}$. For this wavelength the penetration depth is about $300 \mathrm{~nm}$ and so, the scattered light essentially comes from the top Si layers. In this and the following figures the intensity of the spectra is normalized to the maximum value. The spectra obtained in both SIMOX samples show a shift towards lower frequencies and a symmetric broadening in relation to that obtained in bulk $\mathrm{Si}$. This indicates that these layers are under a tensile strain. The symmetrical shape of the lines confirms in both cases the crystalline nature of the top $\mathrm{Si}$ layers, and their broadening indicates the existence of a distribution of strain. No amorphous-like bands are observed. Moreover, for all excitation powers the SS sample shows a shift and a broadening of the Raman peaks bigger than the SIA one, as can be seen in Table II. According to this, there is a higher tensile strain in the surface region of the SS structure in relation to the SIA one.

The results obtained using excitation wavelengths of $488 \mathrm{~nm}$ (penetration depth about $600 \mathrm{~nm}$ ) and $514 \mathrm{~nm}$ (penetration depth about $800 \mathrm{~nm}$ ) show a different behavior. For these wavelengths the substrate under the buried oxide is reached by the scattering volume. In Fig. 6 are plotted the spectra measured for a wavelength of $514 \mathrm{~nm}$ and a power of $1 \mathrm{MW} / \mathrm{cm}^{2}$. In this case, and for both excitation wavelengths, the higher shifts and broadenings take place in the SIA structure. The data obtained for the different powers are listed in Tables III and IV. The increase in the shift and the broadening of the Raman lines from the SIA samples when the penetration depth increases seem to indicate the existence of a significant strain located in the second region of the SIA structure, where

TABLE II. Shifts and broadenings of the Raman lines from the SIMOX samples in relation to bulk Si. Excitation wavelength $457.9 \mathrm{~nm}$. The data are in units of $\mathrm{cm}^{-1}$.

\begin{tabular}{lccccccc}
\hline \hline$P\left(\mathrm{MW} / \mathrm{cm}^{2}\right)$ & \multicolumn{2}{c}{0.25} & \multicolumn{2}{c}{0.5} & \multicolumn{2}{c}{1} \\
\hline Sample & $w$ & $\Delta \Gamma$ & $w$ &.$\Delta \Gamma$ & $w$ & $\Delta \Gamma$ \\
SS & 0.4 & 2.15 & 1 & 4.37 & 1.7 & 4.97 \\
SIA & $\ldots$ & $\ldots$ & 0.8 & 2.68 & 1.1 & 2.83 \\
\hline \hline
\end{tabular}

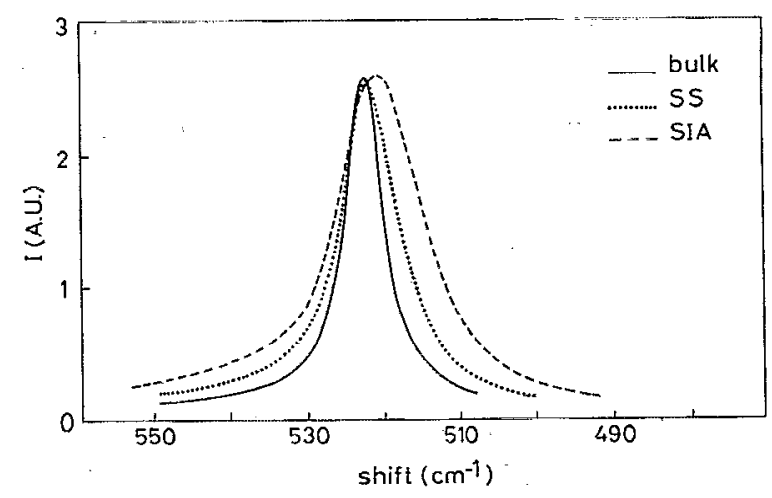

FIG. 6. First-order Raman spectra obtained from the different samples with an excitation power of $1 \mathrm{MW} / \mathrm{cm}^{2}$ and wavelength $514 \mathrm{~nm}$.

the precipitates appear. So, as the penetration depth increases, the contribution to the spectra of this region with precipitates and dislocations increases. Moreover, the spectra obtained for this structure show a small contribution to the higher frequencies side, as can be seen in Fig. 7. This contribution indicates the presence in the SIA sample of a compressive stress. In general, the spectra measured in both samples and for all the excitation wavelengths show an increase of the shift and the broadening when the excitation power increases, indicating an increase of the strain effects with the temperature gradient.

Although we have ascribed the origin of the shifts and broadenings of the Raman peaks to the presence of strain in the layers, these changes in the spectra could also be due to other effects, such as finite crystallite size ${ }^{14}$ or free carrier and doping effects. ${ }^{15}$ However, the spectra obtained at low laser excitation powers are not consistent with the presence of small crystallites, which would give rise to a Raman band in the low frequency side of the first-order Raman silicon band. Then, the influence of the crystallite effect would be rather related to the thermally induced stresses at the crystallite boundaries. Nevertheless, the presence of such microcrystals has not been observed by higher resolution XTEM observations, in agreement with previous works. ${ }^{16,17}$

In relation to the free carrier effects, it is true that a phonon softening can be produced by high carrier concentrations, but the carrier concentrations needed to obtain the changes experimentally observed ought to be very high. Free carrier effects or doping effects do not agree with the spreading resistance measurements carried out in our specimens, which showed a very high resistivity in the top $\mathrm{Si}$

TABLE III. Shifts and broadenings of the Raman lines from the SIMOX samples in relation to bulk Si. Excitation wavelength $488 \mathrm{~nm}$. The data are in units of $\mathrm{cm}^{-1}$.

\begin{tabular}{lcccc}
\hline \hline$P\left(\mathrm{MW} / \mathrm{cm}^{2}\right)$ & \multicolumn{3}{c}{0.25} & \multicolumn{2}{c}{0.5} \\
\hline Sample & $w$ & $\Delta \Gamma$ & $w$ & $\Delta \Gamma$ \\
SS & 0.2 & 1.67 & 0.8 & 1.77 \\
SIA & 0.5 & 2.28 & 1.2 & 3.8 \\
\hline
\end{tabular}


TABLE IV. Shifts and broadenings of the Raman lines from the SIMOX samples in relation to bulk Si. Excitation wavelength $514 \mathrm{~nm}$. The data are in units of $\mathrm{cm}^{-1}$.

\begin{tabular}{lcccccc}
\hline \hline$P\left(\mathrm{MW} / \mathrm{cm}^{2}\right)$ & \multicolumn{2}{c}{0.25} & \multicolumn{2}{c}{0.5} & \multicolumn{2}{c}{1} \\
\hline Sample & $w$ & $\Delta \Gamma$ & $w$ & $\Delta \Gamma$ & $w$ & $\Delta \Gamma$ \\
SS & 0.4 & 1.05 & 0.4 & 1.71 & 0.5 & 3.15 \\
SIA & $\ldots$ & 2.94 & 0.5 & 4.41 & 1.5 & 6.93 \\
\hline \hline
\end{tabular}

layer (almost in the high resistance limit of our spreading resistance system, model SSM-130). In addition to this, the results obtained in bulk silicon clearly rule out the hypothesis of high density generation of carriers by the laser at the powers used in our measurements. Therefore, the main changes observed in the Raman spectra have to be essentially due to strain effects, which are very sensitive to the laser induced temperature gradient in the scattering volume. $^{18-20}$

Different authors ${ }^{4,8,13}$ have reported the existence of a tensile strain in the top silicon layer of as-implanted SS SIMOX structures, as well as a compressive strain below the buried oxide, due to the oxidation reaction which takes place during the implantation. Olego et al. ${ }^{13}$ have observed a recovery of the strain present in the top Si layer with HTA treatments. They did not find shifts of broadenings of the Raman peaks of layers lamp annealed at $1405^{\circ} \mathrm{C}$ for 30 min. According to their observations, and in agreement with the observations of Takahashi et al., ${ }^{12}$ they ascribed the origin of the strain in the layers to the presence of oxide precipitates. In our case, the frequency shifts are similar to those reported by these authors. However, the correlation between the Raman measurements and XTEM observations has allowed us to detect the existence of a higher strain in the surface region of the SS structure, which is free of $\mathrm{SiO}_{2}$ precipitates, in relation to the SIA sample: These results indicate the existence of a stress component not directly related to the precipitates, which depends on the temperature gradient in the sample. In fact, part of the

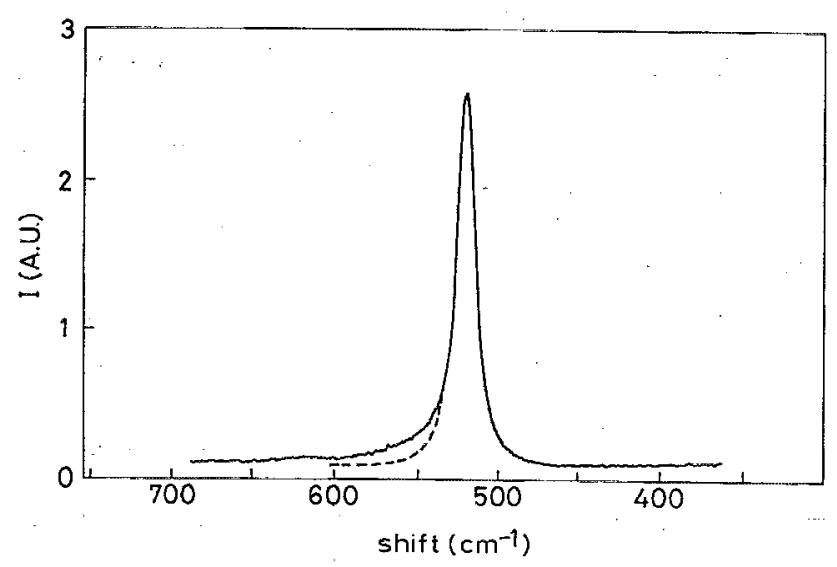

FIG. 7. First-order Raman spectrum obtained from SIA sample, showing a small contribution in the higher frequency side (excitation power 1 $\mathrm{MW} / \mathrm{cm}^{2}$ and wavelength $514 \mathrm{~nm}$ ). The dashed line in the higher frequency side corresponds to a theoretical symmetric peak.

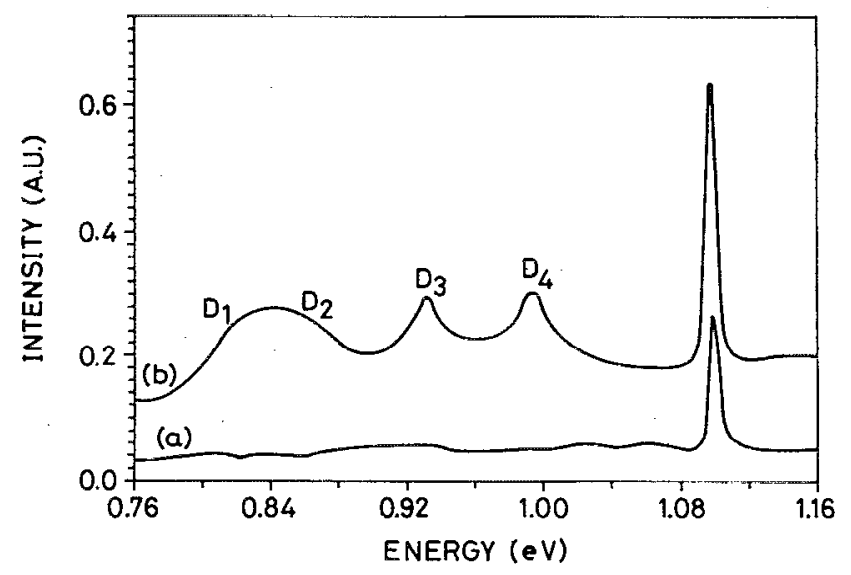

FIG. 8. PL spectra obtained from samples SS (curve a) and SIA (curve b). Excitation wavelength $514 \mathrm{~nm}$.

remaining strain observed in the surface region of the SIA structure would be due to some contribution of the precipitates region, partly included in the scattering volume. Hence, the SIA process would allow to obtain a better recovery of this strain in the top $\mathrm{Si}$ layer free of precipitates, indicating the better quality of this region in relation to the SS structure, with higher mobility values (closer to those of bulk silicon) to be expected. However, for higher penetration depths, the component of the stress due to the precipitates determines a higher strain in the SIA sample. The small compressive strain component observed in the spectra could be due to the existence of a compressive strain in the silicon below the buried oxide (similar to that observed in as-implanted structures ${ }^{4}$ ) or maybe in the silicon regions below the precipitates.

The higher quality of the surface region from SIA structures has been corroborated by photoluminescence measurements. ${ }^{21}$ Figure 8 shows the PL spectra obtained from samples SS (curve a) and SIA (curve b) exciting with a wavelength of $514 \mathrm{~nm}$. Both curves show a peak located in the near band-gap region which is due to the recombination of intrinsic excitons. The intensity of this peak in the SIA sample is a factor of 2 greater than in the SS one, indicating a larger number of nonradiative defects in the single implant material, which is in agreement with the lower strain observed in the top Si layer of the SIA material.

Moreover, the spectrum from the SIA sample shows at lower energies the presence of broad defect related peaks labelled $D_{1}, D_{2}, D_{3}$, and $D_{4}$. Their positions fall within the range of energies previously reported by Duncan et al., ${ }^{22}$ namely, 0.802 to $0.814 \mathrm{eV}, 0.862$ to $0.875 \mathrm{eV}, 0.930$ to $0.948 \mathrm{eV}$ and 0.992 to $1.000 \mathrm{eV}$. The broadest peak, which appears at the lowest energy, includes the $D_{1}$ and $D_{2}$ bands. These peaks were first observed in plastically deformed silicon, and are believed to be related to dislocations. ${ }^{22}$ They have been observed also in bulk silicon in which oxygen is known to have precipitated and in SS SIMOX material. ${ }^{9,22,23}$ In this latter case, the width of the $D$ lines was found to be much broader than in the plastically de- 
formed samples. Drozdov et al. ${ }^{24}$ have reported that a high oxygen concentration can increase considerably the width of the $D$ lines. Weber et al. ${ }^{9}$ have attributed this broadening of the $D$ lines to inhomogeneous strains in the layers, determined by the presence of oxygen precipitates. According to this, the broad bands from the SIA samples (curve b) would be due to excess oxygen in these samples, maybe as oxygen precipitates. The appearance of the $D$ bands in the PL spectrum is consistent with the observation by XTEM of dislocations in the second region of the SIA structure pinned to the precipitates.

This interpretation is supported by the evolution of the PL spectra with the excitation wavelength, normalized to the experimental conditions. The main difference expected between the spectra obtained with the different excitation wavelengths has to be due to the contribution of recombination from below the oxide layer. For the lower excitation wavelength, photoluminescence essentially comes from the top silicon layer, while for the higher ones it comes from both above and below the buried oxide. The PL spectra obtained exciting with the lower wavelength show in both cases a decrease of the intensity of the intrinsic exciton peak. This seems to suggest a higher quality of the material below the buried oxide, when compared to the top silicon film. Anyway, in all the cases the intensity of this peak from the SIA sample is greater than from the SS one. Moreover, the intensity of the $D$ bands remains almost constant, when normalized to the photons flux. In this case, and taking into account the possible diffusion of the carriers, the contribution to the spectra of recombination taking place in the top silicon layer has to remain almost constant. So, this behavior suggests that the origin of these peaks is effectively located in the top Si layer.

\section{CONCLUSIONS}

Although the samples studied do not correspond to optimized structures, the analysis carried out has corroborated the potential of the SIA process for obtaining higher quality material. So, from XTEM observations we have deduced the existence in both cases of a low defect density Si surface layer. The characterization of the samples by Raman scattering and photoluminescence spectroscopy indicates the better quality of this surface region from the structure obtained by sequential implant and anneal, showing a lower strain and a lower concentration of defects acting as nonradiative recombination centers, in relation to the SS structure. Moreover, XTEM images also show the higher quality of the buried oxide layer from SIA struc- tures, which appears free of $\mathrm{Si}$ islands. Therefore, all the experimental results point to the fact that, in spite of the features related to the interface region between the top $\mathrm{Si}$ and the buried oxide layers, better quality material is obtained by the SIA method in comparison with the SS one.

Also, the analysis performed has confirmed the value of Raman scattering and photoluminescence spectroscopy techniques for the characterization of SOI structures. Particularly in the case of Raman scattering, the spectra obtained with different excitation powers are highly sensitive to the structural characteristics of the different layers, as well as to the presence of defects such as dislocations or precipitates. Measurements performed with different excitation wavelengths (and, so, different penetration depths) allow us to characterize the different regions of the structure.

${ }^{1}$ J. C. Sturm, C. K. Chen, L. Pfeiffer, and P. L. F. Hemment, eds., Mater. Res. Soc. Symp. Proc. 107, (1988).

${ }^{2}$ J. P. Colinge, IEEE Elec. Dev. Lett. 9, 97 (1988).

${ }^{3}$ C. Jaussaud, J. Margail, J. Stoemenos, and M. Bruel, Mater. Res. Soc. Symp. Proc. 107, 17 (1988).

${ }^{4}$ A. H. Van Ommen, Nucl. Instrum. Meth. Phys. Res. B 39, 194 (1989).

${ }^{5}$ D. Hill, P. Fraundorf, and G. Fraundorf, J. Appl. Phys. 63, 4933 (1988).

${ }^{6}$ T. F. Cheek and D. Chen, Mater. Res. Soc. Symp. Proc. 107, 53 (1988).

${ }^{7}$ J. Margail, J. Stoemenos, C. Jaussaud, and M. Bruel, Appl. Phys. Lett. 54, 526 (1989).

${ }^{8}$ G. Harbecke, E. F. Steigmeier, P. L. F. Hemment, and K. J. Reeson, Semicond. Sci. Technol. 2, 687 (1987).

${ }^{9}$ J. Weber, H. Baumgart, J. Petruzzello, and G. K. Celler, Mater. Res. Soc. Symp. Proc. 107, 117 (1988).

${ }^{10}$ IBIS Technology Inc., Cherry Hill Drive, Danvers, MA-01923, USA.

${ }^{11}$ A. Romano, J. Vanhellemont, H. Bender, and J. R. Morante, Ultramicroscopy 31, 183 (1989).

${ }^{12}$ J. Takahashi and T. Makino, J. Appl. Phys. 63, 87 (1988).

${ }^{13}$ D. J. Olego, H. Baumgart, and G. K. Celler, Appl. Phys. Lett. 52, 483 (1988).

${ }^{14}$ M. Ingels, M. Stutzmann, and S. Zollner, Mater. Res. Soc. Symp. Proc. 164, 229 (1990).

${ }^{15}$ D. Olego and M. Cardona, Phys. Rev. B 23, 6592 (1981).

${ }^{16}$ B. Y. Mao, P. H. Chang, H. W. Lam, B. W. Shen, and J. A. Keenan, Appl. Phys. Lett. 48, 794 (1986).

${ }^{17} \mathrm{~J}$. Stoemenos, C. Jaussaud, M. Bruel, and J. Margail, J. Cryst. Growth 73, 546 (1985)

${ }^{18}$ J. Raptis, E. Liarokapis, and E. Anastassakis, Appl. Phys. Lett. 44, 125 (1984).

${ }^{19}$ E. Liarokapis and E. Anastassakis, Phys. Scr. 38, 84 (1988).

${ }^{20}$ E. Liarokapis and E. Anastassakis, J. Appl. Phys. 63, 2615 (1988).

${ }^{21}$ A. Pérez, J. Samitier, A. Cornet, J. R. Morante, P. L. F. Hemment and K. P. Homewood, Appl. Phys. Lett. 57, 2443 (1990).

${ }^{22}$ W. M. Duncan, P. H. Chang, B. Y. Mao, and C. E. Chen, Appl. Phys. Lett. 51, 773 (1987).

${ }^{23}$ S. T. Davey, J. R. Davis, K. J. Reeson, and P. L. F. Hemment, Appl. Phys. Lett. 52, 465 (1988).

${ }^{24}$ N. A. Drozdov, A. A. Patrin, and V. T. Tkachev, Phys. Status Solidi A 64, K63 (1981). 